\title{
Towards Improving Recommender System: A Social Trust-Aware Approach
}

\author{
Naziha Abderrahim, Sidi Mohamed Benslimane \\ EEDIS Laboratory, Djillali Liabes University of Sidi Bel Abbes, Sidi Bel Abbes, 22000, Algeria \\ Email: \{naziha.abderahim, benslimane\}@univ-sba.dz
}

\begin{abstract}
Recommender systems have shown great potential to help users find interesting and relevant Web service (WS) from within large registers. However, with the proliferation of WSs, recommendation becomes a very difficult task. Social computing seems offering innovative solutions to overcome those shortcomings. Social computing is at the crossroad of computer sciences and social sciences disciplines by looking into ways of improving application design and development using elements that people encounter daily such as social networks, trust, reputation, and recommendation. In this paper, we propose a social trust-aware system for recommending Web services (WSs) based on social qualities of WSs that they exhibit towards peers at runtime, and trustworthiness of the users who provide feedback on their overall experience using WSs. A set of experiments to assess the fairness and accuracy of the proposed system are reported in the paper, showing promising results and demonstrating that our service recommendation method significantly outperforms conventional similarity-based and trust-based service recommendation methods.
\end{abstract}

Index Terms-Web Service, Social Network, Social Trust, Recommendation.

\section{INTRODUCTION}

Web 2.0 is the second generation of Internet based services that emphasizes the role of users on the Web. With the rapid growth in the number of available services, recommending suitable services to users becomes increasingly important. Recommender systems constitute a significant response to the information overload problem that users have to deal with constantly. These systems rely primarily on users' ratings of items' descriptions. The quality of recommendations is dependent on the nature of details (in terms of quality and quantity) available to users. Collaborative Filtering (CF), which tends to identify similar users to a user, is the dominant technique for recommender systems. However, it is difficult to compute similarity between users when ratings are sparse [1]. Moreover, similar does not always mean trustworthiness, which raises concerns over the reliability of $\mathrm{CF}$ recommendations.

In the context of Web-based Social Networks (SNs) where users are connected together, we need to consider additional factors that can help measure and interpret the similarity between user profiles. Most of existing recommender systems incorporates social trust relationships among users into WSs' recommendation [2]. SNs capture relationships between users like friendship and collegiality. These SNs makes ideal for improving recommender system's capabilities. Furthermore, research in the area of recommender systems in SNs has shown that users would prefer to receive recommendations from whom they trust more. Embedding trust into SNs should lead into trust-aware recommender systems [3]. Hence, recommendations are made based on the ratings given by users; these latters can be either directly or indirectly trusted by the current user.

The proliferation of WSs reflects the second evolution wave of the Internet making the Web a platform for online applications collaboration [4]. However, several constraints continue to limit WSs adoption by the IT community, for instance WSs only know about themselves, not about their users' social connections or peers, they cannot also delegate their invocations to others peers, limit user's intervention considerably, and operate as black boxes [5]. Social computing seems offering innovative solutions to some of these limitations, which improves the quality of recommendations. Social computing is at the crossroad of computer sciences and social sciences disciplines by looking into ways of improving application design and development using elements that people encounter daily. Blending social computing with service-oriented computing leads to Social Web Services (SWSs) that exhibit certain Social Qualities of Service (SQoS) towards peers, thus allows them "knowing" with whom they have worked in the past and with whom they would potentially like to work in the future. Thereby, SWSs are expected to take the initiative in advising users how to develop and reuse value-added services [5].

Building upon these considerations, we propose a recommender system that combines trust-based $\mathrm{CF}$ techniques and social elements so that personalized WSs recommendations are provided.

The paper is organized as below. Section 2 discusses some related work. Section 3 introduces the social trustaware recommendation system. Next implementation and experiments details are described in section 4. Finally, the paper ends with concluding remarks and future work.

\section{RELATED WORK}


The work presented in [6] rely on the generated knowledge from interactions between users and WSs (and by extension from social environments) to enhance WS composition. They propose a Social Composer (SoCo) which handles this issue. SoCo provides recommendations for WSs discovery and selection based on users' interactions and a SN built implicitly from the interactions between users and WSs. The authors in [7] rank WSs using run-time non-functional properties and invocation request. Ranking takes into account the popularity of a WS, considered as a social element and analyzed by users. The work presented in [8] discuss the way the social Web, exemplified by some well-known networking sites like Facebook, contributes to create social applications without having to build SNs. A SN-based framework is proposed in [9] to capture the relationships between WSs, which are substitution, competition, and collaboration, into SNs. The framework permits to achieve two main goals: build WSs' SNs and use these networks to discover WSs. In [10], the authors address the problem of WSs attraction in a SN. Several quality criteria are proposed such as privacy, trust, fairness, and traceability and permit to establish the quality of a SN. SWSs need also to comply with the regulations of the $\mathrm{SNs}$ in which they sign up. This compliance is examined in [11] using commitments. Two types of commitments are identified: social and business. The former connect WSs to SNs. And the latter connect social WSs to composite SWSs. Last but not least, [12] propose an approach that connects isolated WS islands into a global SWS network to enhance the WSs' sociability on a global scale. In [13], a fuzzy-based trust and reputation model using three trust sources is developed to address the problem of WSs discovery. The model takes into account interaction trust, witness reputation, and certified reputation as three input parameters and calculates an overall trust value as output. The authors in [14] integrate social trust into WSs compositions. Trust values are progressively updated over time based on interactions with multiple users. Specifically, they focus on the behavioral (social) aspect of trust in the context of WSs and its role in improving the pragmatics of WS compositions. The work presented in [15] provide a reputation evaluation by integrating a feedback mechanism for the target WSs. The reputation value depends on users' credibility, preferences, and temporal sensitivity. To improve dynamic WS composition, [16] present a framework that on top of functional and non-functional attributes of WSs, they filter and rank solutions based on their trust rating. They establish the trust factor of a WS by measuring the centrality of a service provider and/or a service provider organization in SN. The authors in [17] integrate the social dimension into WSs discovery. They take into account both semantic and structural properties of the service requester. Furthermore, they compute social trust between the service requester and service providers by aggregating three measures: social position, social proximity, and social similarity. The authors argue that when integrating social trust dimension into WSs discovery, this improves wider credibility and acceptance.
A recommender system that can be extended with trustworthiness of users is proposed in [1]. They propose an ontological model of trust between users in a SN to address the limitations of similarity measure in $\mathrm{CF}$ algorithms. Hence, trust can be considered as a measure for expressing the relationship between two users in recommendation systems. A framework for building trust communities using social capital and recommendation system is introduced in [18]. This framework motivates members to share their experiences, feelings, and opinions in an open and honest way with no fear of being judged. They also define an underlying social trust model for SNs, called STrust that separates the interactions in a $\mathrm{SN}$ into two groups (popularity based interactions and engagement based interactions), which enables the model to capture passive interactions such as reading comments without leaving any feedback.

Several works ([19] and [20]) have applied CF to WS recommendation. CF-based WS recommender systems work by collecting user observed QoS records of different WSs and matching together users who share the same information needs or same tastes. However, these works focus only on individual properties (e.g., QoS) of WSs, and do not consider information about personal preferences of QoS on WSs from different users. This is vital for providing personalized WS recommendation. Recently [21] utilized network modeling and analysis methodology to study the trust relationships between users and WSs based on the findings and the WS network model. They propose a CF algorithm called Trust-based Service Recommendation (TSR), which provide users with personalized WS recommendations based on their trust and views on the QoS of desired WSs.

\section{A SOCIAL TRUST-AWARE RECOMMENDATION SYSTEM FOR WSS}

The objective of SNs is to bring people together so they can share experiences, recommendations, advices to cite just a few [18]. As mentioned in [22], trust is a critical factor in the success of SNs adoption. Without trust, members may be reluctant to engage in any form of interaction with peers due to the fears of misjudgment and/or misuse. A SN in which the members trust each other as well as the $\mathrm{SN}$ and service providers is deemed necessary. We call such a SN, a Trusted Social Network (TSN), upon which a Social Trust-aware System for recommending WSs will be built (Fig.1).

\subsection{Identifying Social relationships}

A Social Trust-aware System relies on the interactions between SNs of Users (SNU) and SNs of WSs (SNS) to capture user-to-user, WS-to-WS, and user-to-WS relationships. SNUs and SNSs are graphs that consist of nodes connected to each other through edges.

User-to-User relationships can be social (e.g., when following peers and, establishing friendship connections), and/or informational (e.g., when sharing information, providing recommendation, and gaining access to 
relevant information generated by others).

In this paper, user-to-user relationships are used to derive the degree of trustworthiness between users. Particularly, we consider the quality of social links with peers and the quality of recommendations provided to peers.

\section{A) User's quality of connection}

Social links demonstrate specific affinity between users. User $u$ can have either a strong or a weak affinity with user $v$ based on a personal relationship (e.g., kinship and friendship) or business relationship (e.g., partnership and membership). Models that allow users to create and connect statements about who they know are typically based on the concepts of "Web of trust" or Friend-Of-AFriend (FOAF). A FOAF schema is an RDF vocabulary that a user can use to describe information about himself, such as name, email address, and homepage, as well as information about people he knows. We extend foaf: Person so that users indicate a level of affinity with people they know. This level between $u$ and $v$, denoted by $U_{u, v}^{L O A}$, is specified on a scale of 0 (weak affinity) to 1 (strong affinity). To infer user's affinity with unknown peers, we use the computational model of trust proposed by [23].

Equation (1) describes how transitive affinity values are inferred using a weighted average over all neighbors.

$$
U_{u, v}^{L O A}=\frac{\sum_{p=0}^{n}\left\{\begin{array}{ll}
\left(U_{p, v}^{L O A} * U_{u, p}^{L O A}\right) & \text { if } U_{u, p}^{L O A} \geq U_{p, v}^{L O A} \\
\left(U_{u, p}^{L O A}\right)^{2} & \text { if } U_{u, p}^{L O A}<U_{p, v}^{L O A}
\end{array}\right\}}{\sum_{p=0}^{n} U_{u, p}^{L O A}}
$$

Where $u$ has $n$ neighbors with a paths to $v$.

\section{B) User's quality of recommendation}

The Quality of Recommendation, denoted $U_{u, v}^{Q O R}$, indicates the degree of satisfaction of $v$ regarding recommendations of $u$. This allows detecting trustful recommenders who have a history of making reliable recommendations. Equation (2) calculates $U_{u, v}^{Q o R}$ :

$$
U_{u, v}^{Q o R}=\frac{R R_{u, v}}{T R_{u}}
$$

Where $R R_{\mathrm{u}, \mathrm{v}}$ represents the total number of reliable recommendations that $u$ provides to $v$, and $T R_{\mathrm{u}}$ represents the total number of recommendations that $u$ has provided on the SNU.

\section{C) User's quality of trust}

One fundamental issue in trust-based $\mathrm{CF}$ is how to assess the degree of trustworthiness between users. To calculate the Quality of Trust between $u$ and $v$, denoted $U_{u, v}^{Q o T}$, we use their social connections and interactions. It is measured by (3):

$$
U_{u, v}^{Q o T}=\gamma \times U_{u, v}^{Q o R}+(1-\gamma) \times U_{u, v}^{Q o C}
$$

Where $\gamma$ is a weighting coefficient such as $0 \leq \gamma \leq 1$.
In a SNS, WSs are in constant interactions. New relationships can be formed and existing ones may disappear or change. SN analysis can help WSs take advantage of previous composition scenarios in which they participated, so they can build relationships with other peers that are in these compositions. QoS is employed widely to represent the non-functional properties of WSs (e.g., price, availability, and response time), and has been a key factor in their selection [19].

In this research, we consider SQoS as social properties that characterize the behaviors that WSs exhibit towards peers at run time. In the following, we adopt cooperation, selfishness, and honesty social properties as per [24].

\section{Definition (Cooperation)}

The cooperation quality of a WS refers to the number of times it is solicited by peers for participation in compositions. More the WS is solicited, better is the cooperation quality. To evaluate the cooperation quality denoted as $\operatorname{Coop}_{i, j}$ of WS $i$ versus WS $j$, we track the number of times that WS $j$ invokes WS $i$ in joint compositions.

$$
\operatorname{Coop}_{i, j}=\frac{\sum J C_{i ; j}}{\sum T P_{i, j}}
$$

Where $\sum J C_{i, j}$ is the total number of participations of WS $i$ and $\mathrm{WS} j$ in joint compositions and $\sum T P_{i, j}$ is the Total number of Participations of WS $i$ in compositions.

\section{Definition (Selfishness)}

A WS behaves in a selfish way if it does not show a positive attitude towards others peers. For example, a selfish WS continuously receives positive responses from peers when it seeks their assistance for substitution, but it does continuously the opposite, i.e., declines others' assistance requests [5].

To evaluate the selfishness quality denoted $\operatorname{Sel}_{i, j}$ of WS $i$ versus WS $j$, we track the number of times that WS $i$ declines the request of WS $j$ in a collaboration $\mathrm{SN}$.

$$
\operatorname{Sel}_{i, j}=\frac{\sum D R_{i, j}}{\sum J C_{i, j}}
$$

Where $\sum D R_{i, j}$ is the number of requests of WS $i$ that WS $j$ declines

\section{Definition (Honesty)}

Honesty of WS $i$ is the difference between the advertised or agreed upon QoS parameters values and the actual observed values of these QoS parameters. Equation (6) evaluates honesty quality denoted $\mathrm{H}_{i, j}$ :

$$
\mathrm{H}_{i, j}=1-\frac{\sum\left|A Q o S_{j}^{i}-O Q o S_{j}^{i}\right|}{k}
$$

Where $A Q o S_{j}^{i}$ is the value of Advertised QoS of WS $i$ for WS $j, O Q o S_{j}^{i}$ is the value of Observed QoS of WS $i$ by $\mathrm{WS} j$, and $k$ is the number of times that WS $j$ has invoked WS 
In a SN, people communicate with friends and others in the chain of friends, and share their experiences and opinions within the network about an item such as WS. An opinion has a great impact on members in a SN. However, the trustworthiness of those of whose provide opinions raises different concerns. Indeed, an opinion could be biased because of a recent unsuccessful experience.

In this work, we treat users' opinion ratings for WSs as a measure that assesses to what extent users trust WSs. Hence, to measure user's perception and personalized preference on multiple qualities of a WS including QoS and SQoS, we define (7):

$$
\begin{gathered}
O p_{i}^{u}=\lambda \times\left(\sum_{t}^{N_{q}} W_{q t} \times R a t_{q t}^{u, i}\right)+(1-\lambda) \\
\left(\sum_{l}^{N_{s q}} W_{q l} \times R a t_{q l}^{u, i}\right)
\end{gathered}
$$

Where $\lambda$ is a weighting coefficient such as $0 \leq \lambda \leq 1$, $W_{q t}$ is the preference weight on quality $t$ which ranges from 0 to $1, N q$ is the number of QoS properties of WS $\mathrm{s}, R_{a t}^{u, i}$ which ranges from 1 to 5 , is calculated by averaging all the rating of WS $i$ from user $u$ over the QoS $t$ during all the interactions between $u$ and the WSs.

Likewise, $W_{q l}$ is the preference weight on SQoS $l$ which ranges from 0 to $1, N s q$ is the number of SQoS WS $i, R_{a t}^{u, i}$ which ranges from 1 to 5 , is calculated by averaging all the rating of WS $i$ from $u$ on the social quality $l$ during all the interactions between $u$ and WS $i$.

\subsection{Social trust networks}

Many trust definitions exist depending on the discipline such as sociology [25], psychology [26], economics [22], and computer sciences [27]. In general, trust is a measure of confidence that an entity will behave as expected despite the lack of ability of monitoring this entity [28]. Many definitions are limited to a discipline and may not be directly applicable to SNs. Therefore, it is important to also look at trust from the point of view of SNs.

\subsubsection{Trusted SN of users}

A Trusted SN of Users (TSNU) is a directed graph $T S N U(V ; E)$ where a vertex $u \in V$ represents a user and an edge $e(u ; v) \in E$ denotes the trustworthiness of $v$ in $u$. Computing the trust value between $u$ and $v$ amounts to computing the similarity between them. We use the Pearson Correlation Coefficient (PCC) to compute the similarity between $u$ and $v$ as per (8):

$$
\begin{aligned}
& \operatorname{Sim}_{u, v} \frac{2 \times\left|I_{u} \cap I_{v}\right|}{\left|I_{u}\right|+\left|I_{V}\right|} \times \\
& \frac{\sum_{s \in I_{u} \cap I_{v}}\left(O p_{i}^{u}-\overline{O p_{u}^{u}}\right)\left(O p_{i}^{v}-\overline{O p^{v}}\right)}{\sqrt{\sum_{s \in I_{u} \cap I_{v}}\left(o p_{i}^{u}-\overline{O p^{u}}\right)^{2}} \sqrt{\sum_{s \in I_{u} \cap I_{v}}\left(O p_{i}^{v}-\overline{O p^{v}}\right)^{2}}}
\end{aligned}
$$

Where $\left|I_{u} \cap I_{v}\right|$ is the number of common WSs that are invoked by both $u$ and $v$ and $\left|I_{u}\right|$ and $\left|I_{V}\right|$ are respectively the numbers of WSs invoked by $u$ and $v$. The produced PCC value is within the range of $[-1,1]$, where a higher value represents a higher similarity. $I_{u} \cap I_{v}$ is the set of common WSs that are co-invoked by $u$ and $v, O p_{i}^{u}$ measures $u$ 's perception and personalized preference on multiple qualities of $s$ including QoS and social qualities, $\overline{O p^{u}}$ and $\overline{O p^{v}}$ represent the average QoS and SQoS values of all WSs that $u$ and $v$ have perceived respectively.

\subsubsection{Trusted SN of WSs}

A Trusted SN of WSs (TSNS) is represented with a couple $\operatorname{TSNS}=(S, D)$, where $S$ is a set of nodes representing WSs, $D$ is a set of directed edges which stand for trust relationship between WSs. Each relationship is pondered with a weight that indicates the trust values between two WSs.

Before building a TSN, we compute the trust value between WSs, based on WSs similarity. The main idea is to first isolate the users who have rated both WSs and then apply a similarity computation technique to determine the similarity $\operatorname{Sim} S_{i, j}$ [29]. The similarity between $W S i$ and $W S j$ is measured by computing the PCC:

$$
\begin{gathered}
\operatorname{Sim}_{i, j}=\frac{2 \times|i \cap j|}{|i|+|j|} \times \\
\frac{\sum_{u \in i \cap j}\left(o p_{i}^{u}-\overline{O p_{l}}\right)\left(o p_{j}^{u}-\overline{O p_{J}}\right)}{\sqrt{\sum_{u \in i \cap j}\left(O p_{i}^{u}-\overline{O p_{l}}\right)^{2}} \sqrt{\sum_{u \in i \cap j}\left(o p_{j}^{u}-\overline{O p_{J}}\right)^{2}}}
\end{gathered}
$$

Where $i \cap j$ denotes the set of users who rated both $W S i$ and $W S j ; O p_{i}^{u}$ and $O p_{j}^{u}$ are the opinion values of $u$ on $W S i$ and $W S j$; and $\overline{O p_{\imath}}$ and $\overline{O p_{j}}$ are the average ratings of the $W S i$ and $W S j$ by those users respectively, $|i \cap j|$ is the number of users who co-invoke WS $i$ and $W S j ;|i|$ and $|j|$ are the numbers of users that invoke $W S i$ and $W S j$, respectively.

\subsection{Social Trust-aware system for recommending WSs}

In our social trust-aware recommender system, we combine user-based and WS-based collaborative filtering to calculate trust value from users' and WSs' perspectives.

The user-based trust value of a WS $i$ for a target user $u$, denoted $\operatorname{Tr}_{U}^{u, i}$ is computed using (10):

$$
\operatorname{Tr}_{U}^{u, i}=\frac{\sum_{v \in U_{k}} \operatorname{Sim} U_{u, v} \times O p_{i}^{v}}{\sum_{v \in U_{k}} \operatorname{Sim} U_{u, v}}
$$

Where $U_{k}$ denotes the set of top-k most similar users who have rated WS $i$ and have a user's quality of trust with $u$ higher than the threshold $t$ (i.e. $U_{u, v}^{Q o T}>t$ ).

Similarly, we compute the WS-based trust value of $W S i$ for a target user $u$, denoted $\operatorname{Tr}_{S}^{u, i}$ with (11):

$$
T r_{S}^{u, i}=\frac{\sum_{j \in S_{k}} \operatorname{Sim} S_{i, j} \times O p_{j}^{u}}{\sum_{j \in S_{k}} \operatorname{Sim} S_{i, j}}
$$

Where $S_{k}$ denotes the set of top-k most similar WSs that have been rated by $u$ and have SQoS with $W S i$ higher than the threshold $t$ (i.e. $\mathrm{SQoS}_{i, j}>t$ ).

Finally, we provide a unified metric that combine the user-based $\mathrm{CF}$ and WS-based $\mathrm{CF}$ into a hybrid $\mathrm{CF}$ 
recommendation to measure the capability of a recommendation for a target $u$. The balancing the two types of trust values is given by the (12):

$$
\operatorname{Trust}_{i}^{u}=\varepsilon \times \operatorname{Tr}_{U}^{u, i}+(1-\varepsilon) \times \operatorname{Tr}_{S}^{u, i}
$$

Where $0 \leq \varepsilon \leq 1$ is a weighting factor, which can be used to determine the importance of $\operatorname{Tr}_{U}^{u, i}$ and $T r_{S}^{u, i}$.

It is not difficult to see, when $\varepsilon=1$, this equation will reduce to the user-based $\mathrm{CF}$. On the other extreme, when $\varepsilon=0$, this equation will reduce to service-based $\mathrm{CF}$. With an appropriate value of $\varepsilon$, this hybrid $\mathrm{CF}$ will recommend to user the favorite WSs of the users to some extent who are both similar and trustworthy to the target user.

After calculating the trust value of each WS that will be recommended to a user, we obtain a ranked list of WSs according to their trust values. The top-k WSs in the list will be recommended to a user. The overall process of the proposed social trust-aware recommendation system is represented in Algorithm1.

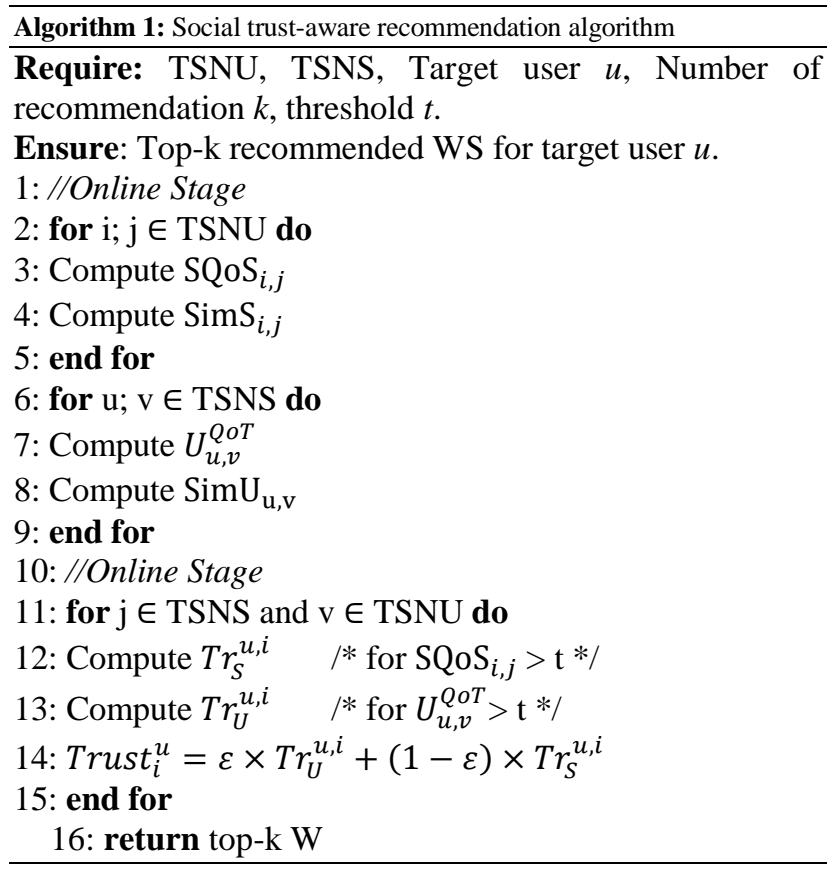

\section{IMPLEMENTATION AND EXPERIMENTS}

To evaluate the performance of our system, we developed a Java-based prototype named Social Trustaware REcommender System for web Services (STRESS). We conduct a set of experiments to evaluate the performance and scalability of STRESS. To demonstrate how the proposed tool works in a specific implementation process, using an AMD Phenom TMII X4 955 machine with 8GB of memory running an Ubuntu 11.04. Fig. 1 shows a screenshot of STRESS.

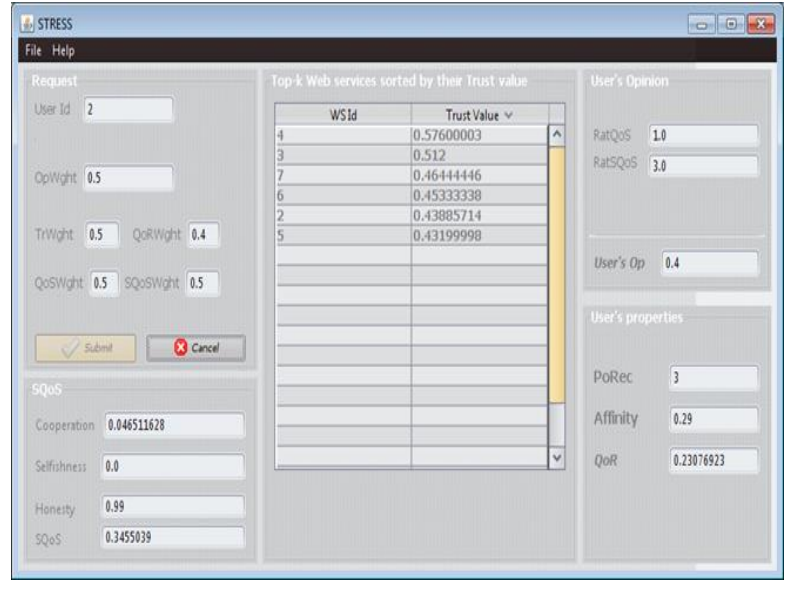

Fig.1. STRESS's main interface

\subsection{Data collection}

To evaluate STRESS's performance, we conducted a series of large-scale simulation experiments based on three real-world dataset from WS-DREAM [30] that capture interactions between users and WSs. The first dataset [31] contains 150 files, where each file includes 10,000 WS invocations on 100 WSs by a service user. Table 1 provides some samples of the results.

Table 1. Samples of the results

\begin{tabular}{|l|c|c|r|r|l|}
\hline ClientIP & $\begin{array}{c}\text { WSI } \\
\text { D }\end{array}$ & $\begin{array}{c}\text { Respo } \\
\text { nse } \\
\text { Time } \\
(\mathbf{m s})\end{array}$ & $\begin{array}{c}\text { Data } \\
\text { Size }\end{array}$ & $\begin{array}{c}\text { HTTP } \\
\text { Code }\end{array}$ & $\begin{array}{c}\text { HTTP } \\
\text { Message }\end{array}$ \\
\hline $\begin{array}{l}35.9 .27 .2 \\
6\end{array}$ & 8451 & 2736 & 582 & 200 & OK \\
\hline $\begin{array}{l}35.9 .27 .2 \\
6\end{array}$ & 8460 & 804 & $\begin{array}{r}1441 \\
9\end{array}$ & 200 & OK \\
\hline $\begin{array}{l}35.9 .27 .2 \\
6\end{array}$ & 8953 & 20176 & 2624 & -1 & $\begin{array}{l}\text { java.net.Socket } \\
\text { TimeoutExcepti } \\
\text { on }\end{array}$ \\
\hline
\end{tabular}

The second dataset ([30] and [32]) contains real-world QoS evaluation results from 339 users on 5,825 WSs. The third dataset [33] contains real-world QoS evaluation results from 142 users invoking 4,532WSs over 64 different time slots.

\subsection{Impact of SQoS on WS recommendation}

The first experiment concerns evaluating the impact of SQoS on WS recommendation i.e. trust value was computed from users' perspective and WSs' perspective respectively. Experiments where accomplished to derive the top-k WSs for a target user ( $\mathrm{Id}=2$ ), according to the three following cases.

- $\quad$ Case 1: $\lambda=0$.

In the first case, we give more importance to SQoS than to QoS (i.e., $\lambda=0$ ). Fig. 2 reports the user-based trust values, the WS-based trust values, and the aggregated trust values for $k=10$.

- $\quad$ Case 2: $\lambda=0.5$.

In the second case, SQoS and QoS have the same degree of importance (i.e., $\lambda=0.5$ ). Fig. 3 reports the 
user-based trust values, the WS-based trust values, and the aggregated trust values for $k=10$.

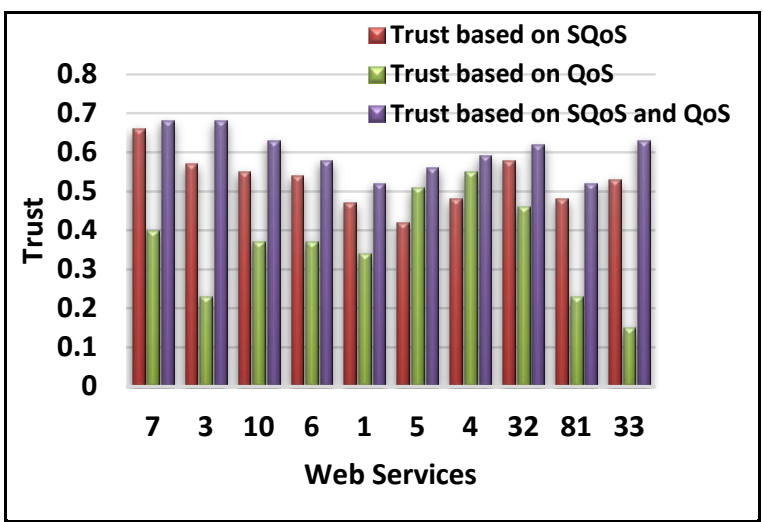

Fig. 2. Impact of SQoS/QoS on WS recommendation

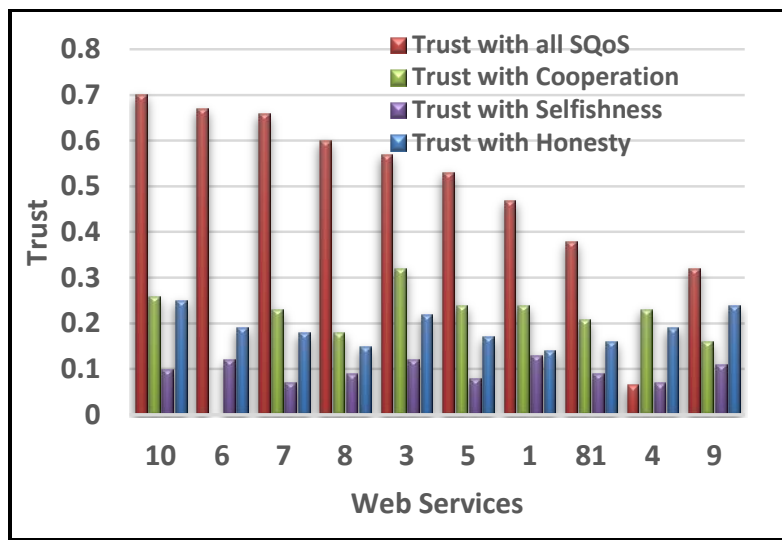

Fig. 3. Impact of individual SQoS on WS recommendation

We can observe from Fig. 3 that the trust value is highly influenced by SQoS, and we have better trust value when we take into account the three aforementioned social qualities.

- $\quad$ Case 3: $\lambda=1$.

In the third case, only QoS is considered for computing the opinion of user (i.e., $\lambda=1$ ). Fig. 4 reports the userbased trust values, the WS-based trust values, and the aggregated trust values for $k=10$.

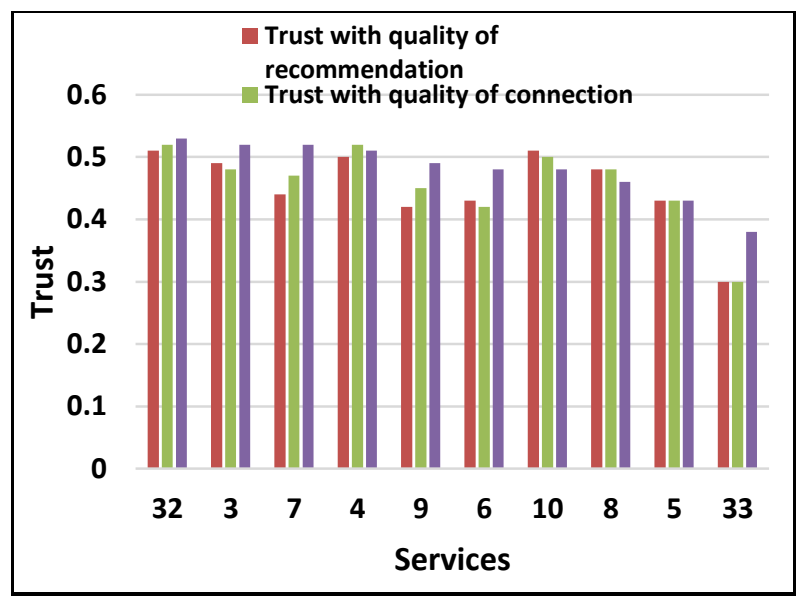

Fig. 4. Impact of user's quality of trust on WSs recommendation
We can observe from Fig. 4 that we obtained best values of trust in almost all WSs, when we take into account the qualities of recommendation and connection.

\subsection{Impact of individual SQoS on WS recommendation}

To evaluate the impact of individual SQoS on calculating trust value from users' perspective and WSs' perspective respectively, we conduct a set of experiments.

Table 2 shows WS's trust value for target user $(\mathrm{Id}=2)$, which was computed based on the variation of the weighting factors of social qualities defined in section III.1. To consider only the SQoS, we have fixed $\lambda$ to 0 .

Table 2. Comparison of trust based on SQoS

\begin{tabular}{|c|c|c|c|c|}
\hline WsId & $\begin{array}{c}\text { Trust } \\
\text { with all } \\
\text { SQoS }\end{array}$ & $\begin{array}{c}\text { Trust }_{\mathbf{i}}^{\boldsymbol{u}} \\
\text { with } \\
\text { Cooperation }\end{array}$ & $\begin{array}{c}\text { Trust }_{\mathbf{i}}^{\boldsymbol{u}} \\
\text { with } \\
\text { Selfishness }\end{array}$ & $\begin{array}{c}\text { Trust }_{\mathbf{i}}^{\boldsymbol{u}} \\
\text { With }_{\text {Honesty }}\end{array}$ \\
\hline 10 & 0.70 & 0.26 & 0.10 & 0.25 \\
\hline 6 & 0.67 & 0.00 & 0.12 & 0.19 \\
\hline 7 & 0.66 & 0.23 & 0.07 & 0.18 \\
\hline 8 & 0.60 & 0.18 & 0.09 & 0.15 \\
\hline 3 & 0.57 & 0.32 & 0.12 & 0.22 \\
\hline 5 & 0.53 & 0.24 & 0.08 & 0.17 \\
\hline 1 & 0.47 & 0.24 & 0.13 & 0.14 \\
\hline 81 & 0.38 & 0.21 & 0.09 & 0.16 \\
\hline 4 & 0.65 & 0.23 & 0.07 & 0.19 \\
\hline 9 & 0.32 & 0.16 & 0.11 & 0.24 \\
\hline
\end{tabular}

4.4. Impact of user's quality of trust on WSs recommendation

To determine the impact of user's quality of trust on WS recommendation, we computed the trust value: (i) with only the quality of recommendation, (ii) with only the quality of connection, (iii) with both qualities: recommendation and connection. Table 3 summarizes the obtained results.

Table 3. User's trust quality values

\begin{tabular}{|c|c|c|c|}
\hline WsId & $\begin{array}{c}\text { Trust } \\
\boldsymbol{U}_{\boldsymbol{i}, \boldsymbol{v}}^{\boldsymbol{u}} \text { with }\end{array}$ & $\begin{array}{c}\text { Trust }_{\boldsymbol{i}}^{\boldsymbol{u}} \text { with } \\
\boldsymbol{U}_{\boldsymbol{u}, \boldsymbol{v}}^{\boldsymbol{Q o \boldsymbol { C }}}\end{array}$ & Trust $_{\mathbf{i}}^{\boldsymbol{u}} \mathbf{w i t h}_{\boldsymbol{u , v}}^{\boldsymbol{Q o T}}$ \\
\hline 32 & 0.51 & 0.52 & 0.53 \\
\hline 3 & 0.49 & 0.48 & 0.52 \\
\hline 7 & 0.44 & 0.47 & 0.52 \\
\hline 4 & 0.50 & 0.52 & 0.51 \\
\hline 9 & 0.42 & 0.45 & 0.49 \\
\hline 6 & 0.43 & 0.42 & 0.48 \\
\hline 10 & 0.51 & 0.50 & 0.48 \\
\hline 8 & 0.48 & 0.48 & 0.46 \\
\hline 5 & 0.43 & 0.43 & 0.43 \\
\hline 33 & 0.30 & 0.30 & 0.38 \\
\hline
\end{tabular}

4.5. Impact of the CF techniques on WSs recommendation

To determine the impact of CF techniques on WS recommendation, we compute the trust value of a WS $i$ for the target user $u$ using:

- User-based CF technique (i.e. $\varepsilon=1$ ).

- WS-based CF technique (i.e. $\varepsilon=0$ ).

- Combination of user-based and WS-based CF techniques (i.e., $\varepsilon=0.5$ )

In Table 4, a comparison between obtained trust values is presented based on three cases. 
Table 4. Comparison between trust values with $\varepsilon=1, \varepsilon=0.5$ and $\varepsilon=0$.

\begin{tabular}{|c|c|c|c|}
\hline WsId & $\begin{array}{c}\text { Trust }_{i}^{u} \\
\text { with } \varepsilon=1\end{array}$ & $\begin{array}{c}\text { Trust }_{i}^{u} \\
\text { with } \varepsilon=0\end{array}$ & $\begin{array}{c}\text { Trust }_{\mathrm{i}}^{u} \\
\text { with } \varepsilon=0.5\end{array}$ \\
\hline 9 & 0.56 & 0.48 & 0.69 \\
\hline 7 & 0.51 & 0.54 & 0.57 \\
\hline 5 & 0.48 & 0.47 & 0.49 \\
\hline 4 & 0.48 & 0.56 & 0.59 \\
\hline 32 & 0.48 & 0.56 & 0.59 \\
\hline 8 & 0.46 & 0.52 & 0.63 \\
\hline 6 & 0.45 & 0.58 & 0.64 \\
\hline 3 & 0.44 & 0.54 & 0.61 \\
\hline 10 & 0.40 & 0.58 & 0.66 \\
\hline 1 & 0.34 & 0.45 & 0.50 \\
\hline
\end{tabular}

From the result of this experiment summarized in Table 4 and the Fig. 5, we conclude that the combination of user-based and WS-based CF recommendation improves significantly the trust value of WS.

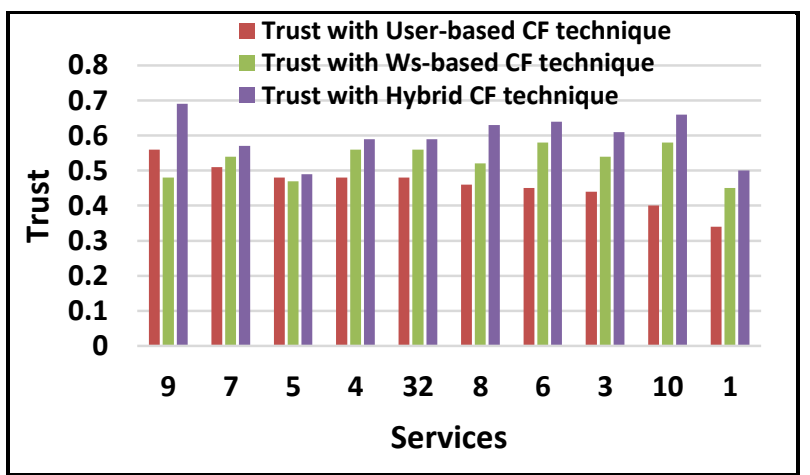

Fig. 5. Impact of the CF techniques on WSs recommendation

From above experimental result, we demonstrate that combining similarity and trust among users and WSs is indeed valuable to WS recommendation. The values of $\lambda$ and $\varepsilon$ have influence on the performance of our method. In this work, when $\lambda=0.5$ and, $\varepsilon=0.5$, our hybrid CFbased recommender system has a considerably better performance than the other cases.

\section{CONCLUSION}

As the social network is growing very fast by doubling the number of people joining every year [23], the possibility of getting a huge number of opinion regarding a particular item is very common. This requires a recommender system to summarize or filter the top opinions or recommendation in terms of quality of the opinion and the trust between the user and the opinion giver [34]. In this paper, we proposed a system for providing recommendation based on the Social Trust Network. The system combines SN of users and SN of WSs to provide recommendations for a target user. The provided recommendations are based not only on the similarity between users and between WSs, but also on the trust value of user and WS. There are a few issues we would like to address in the future. First, we will carry out some researches on trust-aware service composition which different from service recommendation, since it needs to return an optimal composition of multiple services rather than an optimal single service. Second, we plane to evaluate our system with datasets from real-word serviceoriented environments with social networks.

\section{REFERENCES}

[1] Zarghami, A., Fazeli, S., Dokoohaki, N., Matskin, M., 2009. Social trust-aware recommendation system: A tindex approach. In: IEEE (Ed.), Web Intelligence and Intelligent Agent Technologies, WI-IAT'09. Milan, Italy, pp. 85-90, 10.1109/WI-IAT.2009.237.

[2] Tang M., Xu Y., Liu J., Zheng Z., Liu X., 2013: TrustAware Service Recommendation via Exploiting Social Networks. IEEE SCC, pp. 376-383.

[3] Ray, S., 2012. Identifying influential taggers in trust-aware recommender systems. In: IEEE (Ed.), Advances in Social Networks Analysis and Mining (ASONAM'12). Istanbul, pp. 1284-1288.

[4] Dragoni, N., 2010. A survey on trust-based web service provision approaches. In: Dependability (DEPEND), Third International Conference. pp. 83-91, 10.1109/DEPEND.2010.21.

[5] Maamar, Z., Faci, N., Wives, L. K., Yahyaoui, H., Hacid, H., 2011c. Toward a Method for Engineering Social Web Services. In: 4th IFIP WG 8.1 Working Conference on Method Engineering, ME. Paris, France, Volume 351, pp. 153-167.

[6] Maaradji, A., Hacid, H., Daigremont, J., Crespi, N., 2010. Towards a social network based approach for services composition. In: Communications (ICC), IEEE International Conference. Cape Town South Africa.

[7] Wu, Q., Iyengar, A., Subramanian, R., Rouvellou, I., SilvaLepe, I., Mikalsen, T., 2009. Combining quality of service and social information for ranking services. In: 7th International Joint Conference, ICSOC-Service Wave. Sweden, pp. 561-575.

[8] Ko, N. M. Cheek, G. P. S. M., Sandhu, R., 2010. Social network connect services. IEEE Computer 43 (8), 37-43.

[9] Maamar, Z., Faci, N., Badr, Y., Wives, L. K., Benslimane, D., de Oliveira, J. P. M., 2011a. Towards a framework for weaving social networks principles into web services discovery. In: the International Conference on Web Intelligence, Mining and Semantics, WIMS. ACM, Sogndal Norway, pp. 51, 978-1-4503-0148-0/11/05.

[10] Faci, N., Maamar, Z., Ghodous, P., 2012. Which social networks should web services sign-up in? In: AAAI Spring Symposium on Intelligent Web Services Meet Social Computing. Palo Alto, USA, pp.22-26.

[11] Maamar, Z., Faci, N., Luck, M., Hachimi, S., 2012. Specifying and implementing social web services operation using commitments. In: Symposium on Applied Computing (SAC'12). Riva del Garda, Italy, 978-1-45030857-1/12/03.

[12] Chen, W., Paik, I., Hung, P. C., 2013a. Constructing a global social service network for better quality of web service discovery. Services Computing, IEEE Transactions.

[13] Ludwig, S. A., Pulimi, V., Hnativ, A., 2009. Fuzzy approach for the evaluation of trust and reputation of services. In: IEEE International Conference on Fuzzy Systems. pp. 115-120.

[14] Paradesi, S. M., Doshi, P., 2009. Toward integrating social trust into web service compositions. In: AAAI Spring Symposium: Social Semantic Web: Where Web 2.0 Meets Web 3.0. pp. 65-66. 
[15] Malik, Z., Bouguettaya, A., 2009. Rateweb: Reputation assessment for trust establishment among web services. The VLDB Journal 4 (18), 885-911, 10.1007/s00778-0090138-1.

[16] Bansal, S. K., Bansal, A., Blake, M. B., 2010. Trust-based dynamic web service composition using social network analysis. In: IEEE International Workshop on Business Applications of Social Network Analysis. pp. 1-8.

[17] Louati, A., Haddad, J. E., Pinson, S., 2012. Trust-based service discovery in multi-relation social networks. In: 10th International Conference, ICSOC 2012. Shanghai China, pp. 664-671.

[18] Nepal, S., Sherchan, W., Paris, C., 2011. Strust: A trust model for social networks. In: International Joint Conference of IEEE TrustCom -11/IEEE ICESS-11/FCST11. pp. 841-846, 10.1109/TrustCom.2011.112.

[19] Chen, X., Zheng, Z., Liu, X., Huang, Z., Sun, H., 2013b. Personalized qos-aware web service recommendation and visualization. Services Computing, IEEE Transactions 6 (1), 35-47, 10.1109/TSC.2011.35.

[20] Zheng, Z., Ma, H., Lyu, M. R., King, I., 2009. Wsrec: A collaborative filtering based web service recommendation system. In: Web Services, ICWS. IEEE International Conference. pp. 437-444, 10.1109/ICWS.2009.30.

[21] Deng, S., Huang, L., Wu, J., Wu, Z., 2013. Trust-based personalized service recommendation: A network perspective. Journal of computer science and technology 29 (1), pp. 69-80.

[22] Huang, F., 2007. Building social trust: A human-capital approach. Journal of Institutional and Theoretical Economics (JITE) 163 (4), 552-573.

[23] Golbeck, J., 2006. Combining Provenance with Trust in Social Networks for Semantic Web Content Filtering. IPAW pp. 101-108.

[24] Maamar, Z., Faci, N., Wives, L. K., Badr, Y., Santos, P. B., de Oliveira, J. P. M., 2011b. Using social networks for web services discovery. IEEE internet computing 15 (4).

[25] Molm, L. D., Takahashi, N., Peterson, G., 2000. Risk and trust in social exchange: An experimental test of a classical proposition. American Journal of Sociology 5 (105), 13961427.

[26] Cook, K. S., Yamagishi, T., Cheshire, C., Cooper, R., Matsuda, M.and Mashima, R., 2005. Trust building via risk taking: A cross-societal experiment. Social Psychology Quarterly 2 (68), 121-142.

[27] Maheswaran, M., Tang, H. C., Ghunaim, A., 2007, Towards a gravity based trust model for social networking systems. In: 27th International Conference on Distributed Computing Systems Workshops ICDCSW '07.

[28] Singh, S., Bawa, S., 2007. Privacy, trust and policy based authorization framework for services in distributed environments. International Journal of Computer Science 2 (2), 85-92.

[29] Sarwar, B., Karypis, G., Konstan, J., Riedl, J., 2001. Itembased collaborative filtering recommendation algorithms.
In: WWW '01 Proceedings of the 10th international conference on World Wide Web. Hong Kong, pp. 285-295.

[30] Zheng, Z., Lyu, M. R., 2010. Collaborative reliability prediction for service oriented systems. In: Software Engineering, ACM/IEEE 32nd International Conference (ICSE'10). Cape Town, pp. 35-44.

[31] Zheng, Z., Ma, H., Lyu, M. R., King, I., 2011. QoS-aware Web service recommendation by collaborative filtering. Services Computing, IEEE Transactions 4 (2), pp. 140152.

[32] Zhang, Y., Zheng, Z., Lyu, M. R., 2011a. Exploring latent features for memory-based qos prediction in cloud computing. In: the 30th IEEE Symposium on Reliable Distributed Systems (SRDS'11).

[33] Zhang, Y., Zheng, Z., Lyu, M. R., 2011b. Wspred: A timeaware personalized qos prediction framework for web services. In: Proceedings of IEEE Symposium on Software Reliability Engineering (ISSRE'11). Hiroshima, pp. 210219.

[34] Bhuiyan, T., Josang, Audun, \& Xu, Yue (2010) Trust and reputation management in web-based social network. In Usmani, Zeeshan-Ul-Hasan (Ed.) Web Intelligence and Intelligent Agents. InTech, pp. 207-232.

[35] Sumathi, Niranjan N. Chiplunkar, Ashok Kumar A., Dynamic Discovery of Web Services using WSDL. In I.J. Information Technology and Computer Science, 2014, 10, 56-62, 10.5815/ijitcs.2014.10.08.

\section{Authors Profiles}

Naziha Abderrahim is a $\mathrm{PhD}$ student in computer science Department at the University of Sidi Bel-Abbes, Algéria. She received a M.S. degree in computer science in 2010 from the Computer Science Department of Tlemcen University. Her research interests include Web services and social networks, and recommender systems.

Sidi Mohamed Benslimane is an Associate Professor at the Computer Science Department of Sidi Bel Abbes University, Algeria. He received his $\mathrm{PhD}$ degree in computer science from Sidi Bel Abbes University in 2007. He also received a M.S. and a technical engineer degree in computer science in 2001 and 1994 respectively from the Computer Science Department of Sidi Bel Abbes University, Algeria. He is currently Head of Research Team 'Service Oriented Computing' at the Evolutionary Engineering and Distributed Information Systems Laboratory, EEDIS. His research interests include, semantic web, service oriented computing, ontology engineering, information and knowledge management, distributed and heterogeneous information systems and context-aware computing.

How to cite this paper: Naziha Abderrahim, Sidi Mohamed Benslimane,"Towards Improving Recommender System: A Social Trust-Aware Approach", IJMECS, vol.7, no.2, pp.8-15, 2015.DOI: 10.5815/ijmecs.2015.02.02 